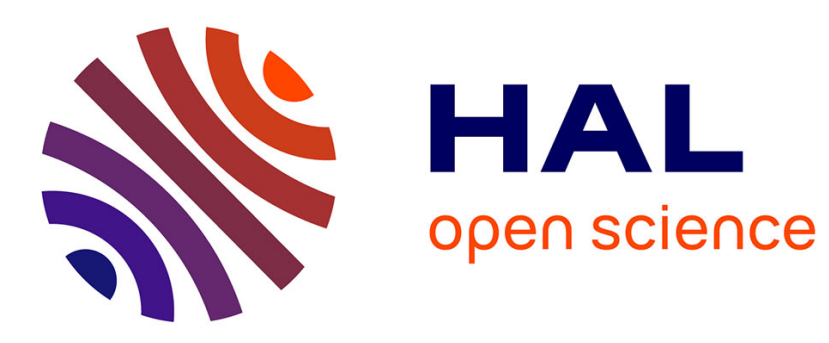

\title{
Understanding Maximum Probability Domains with Simple Models
}

Osvaldo Mafra Lopes Jr., Benoît Braïda, Mauro Causà, Andreas Savin

\section{To cite this version:}

Osvaldo Mafra Lopes Jr., Benoît Braïda, Mauro Causà, Andreas Savin. Understanding Maximum Probability Domains with Simple Models. Advances in the Theory of Quantum Systems in Chemistry and Physics, Springer, pp.173-184, 2011, Progress in Theoretical Chemistry and Physics 978-94-0072075-6. 10.1007/978-94-007-2076-3_10 . hal-01627721

\section{HAL Id: hal-01627721 \\ https://hal.sorbonne-universite.fr/hal-01627721}

Submitted on 21 Nov 2017

HAL is a multi-disciplinary open access archive for the deposit and dissemination of scientific research documents, whether they are published or not. The documents may come from teaching and research institutions in France or abroad, or from public or private research centers.
L'archive ouverte pluridisciplinaire HAL, est destinée au dépôt et à la diffusion de documents scientifiques de niveau recherche, publiés ou non, émanant des établissements d'enseignement et de recherche français ou étrangers, des laboratoires publics ou privés. 


\title{
Understanding Maximum Probability Domains with Simple Models
}

\author{
Osvaldo Mafra Lopes Jr., Benoît Braïda, Mauro Causà, and Andreas Savin
}

\begin{abstract}
The paper presents maximum probability domains (MPDs). These are regions of the three dimensional space for which the probability to find a given number of electrons is maximal. In order to clarify issues hidden by numerical uncertainties, some simple models are used. They show that MPDs reproduce features which one would expect using chemical intuition. For a given number of electrons, there can be several solutions, corresponding to different chemical situations (e.g. different bonds). Some of them can be equivalent, by symmetry. Symmetry can produce, however, alternative solutions. The models show that MPDs do not exactly partition space, and they can also be formed by disjoint subdomains. Finally, an example shows that a partition of space, as provided by loge theory, can lead to situations difficult to deal with, not present for MPDs.
\end{abstract}

\subsection{Introduction}

In the last few years a method was explored which allows to analyze electronic wave functions by describing the regions of space for which the probability to find a given number of electrons, $v$, is maximal [1-11]. When $v=2$, it relates to Lewis' concept of electron pairs and provides thus a connection between quantum mechanics and the traditional way of thinking of chemists. This paper summarizes

O. Mafra Lopes Jr. • B. Braïda • A. Savin ( $\square)$

Laboratoire de Chimie Théorique, CNRS and UPMC Univ Paris 6, 4 place Jussieu, 75252 Paris, France

e-mail: osvaldo.mafra@gmail.com; braida@lct.jussieu.fr; andreas.savin@lct.jussieu.fr

M. Causà

Dip. di Chimica, Universita’ di Napoli Federico II, Via Cintia, 80126 Napoli, Italy

e-mail:mauro.causa@unina.it 
its main features, and illustrates them by using simple quantum mechanical models. These allow to clarify some features which may be blurred by numerical issues in realistic situations.

\subsection{Method}

\subsubsection{Maximal Probability Domains}

For a system in the state described by the wave function $\Psi$, the probability to find $v$ and only $v$ electrons out of $N$ in a three-dimensional region $\Omega$ is given by

$$
p_{v}(\Omega)=\left(\begin{array}{c}
N \\
v
\end{array}\right) \int_{\Omega} d x_{1} \ldots d x_{v} \int_{\bar{\Omega}} d x_{v+1} \ldots d x_{N}\left|\Psi\left(x_{1}, \ldots, x_{N}\right)\right|^{2}
$$

where $\bar{\Omega}$ is the complement of $\Omega, R^{3} \backslash \Omega$, and the binomial coefficient is added to take into account electron indistinguishability. A maximal probability domain, MPD, is a region of space maximizing $p_{v}(\Omega)$. It will be denoted by $\Omega_{v}$.

Please notice that $p_{v}$ is not restricted to ground states, and that $\Omega$ can be formed of disjoint subdomains.

The computation of $p_{v}$, Eq. 10.1, is less difficult as it may seem, at least for certain forms of the wave function. In particular, for a single Slater determinant, one first computes the overlaps of all occupied orbitals over the regions $\Omega$,

$$
S_{i j}(\Omega)=\int_{\Omega} \phi_{i}(x) \phi_{j}(x) d x
$$

Next, the eigenvalues of the matrix with elements $S_{i j}$ are obtained. From them, the probabilities are quickly computed for all $v$, with a recursive formula [3].

The presently running programs use a grid of small cubes. To represent a spatial domain $\Omega$, a collection of such cubes is used. The procedure to optimize $\Omega$ is the following. We first start by guessing a domain, either in a trivial way, like using a larger cube (union of small unit cubes) located in the region of interest, or by using ELF basins [12,13], produced, e.g., with the TopMod program [14]. In the present version of the MPD program, two optimization algorithms are available. In one of them, small cubes are randomly added or deleted, the step being accepted when the probability of the new domain is increased. This algorithm was already used and described in more detail in Ref. [15]. The other algorithm which can be used, takes advantage of the availability of shape derivatives, as described in Ref. [3]. The derivative indicates whether one should add or delete small cubes on the surface of the domain in order to increase the probability. 
Programs to provide MPDs now available can use single determinant wave functions from calculations produced by the Gaussian suite of programs [16] for molecules, or by Crystal-98 [17] for periodic systems. MPDs can be produced also with correlated wave functions, via a Quantum Monte Carlo program, cf. [10, 15]. Probabilities can be computed for multi-determinant wave functions [18], but the optimization of $\Omega$ is not implemented yet.

\subsubsection{Similarities and Differences}

For a well-localized pair of electrons, MPDs provide regions of space which resemble the regions where orbitals can be localized. Notice, however, that localized orbitals extend to infinity, while the MPDs extend over a given region of space. In this respect, they resemble the basins showing up in the Quantum Theory of Atoms in Molecules [19], or in the Electron Localization Function (ELF) approach [12,13]. For single Slater determinants, in the ideal limiting case of strictly localized (nonoverlapping) orbitals, the localization domain of the orbitals, the ELF basins, and the MPDs become identical [20].

The expression for the probability $p_{v}$ may remind of the $v$-particle reduced density matrices. The latter are obtained, however, by integrating $x_{v+1}, \ldots, x_{N}$ over the whole space, not just over $\bar{\Omega}$. In particular, $p_{1}(\Omega)$ is not equal to the integral of the one-particle density over the region $\Omega$,

$$
\int_{\Omega} \rho\left(x_{1}\right) d x_{1}=N \int_{\Omega} d x_{1} \int d x_{2} \ldots d x_{N}\left|\Psi\left(x_{1}, \ldots, x_{N}\right)\right|^{2}
$$

The latter is, in fact, not the probability to find one particle in $\Omega$, but the average number of particles in $\Omega$ [1], the population of $\Omega$. For example, for the dissociated hydrogen molecule in its electronic ground state, the probability to find one electron in the half-space containing one of the protons is equal to 1 , as is the population. For the ionic excited state, $\mathrm{H}^{+} \ldots \mathrm{H}^{-} \leftrightarrow \mathrm{H}^{-} \ldots \mathrm{H}^{+}$, the probability to find one electron in one half-space is zero, while the population is still one.

With population analysis, and also with the valence bond approach, a reference space of atomic functions is defined. Such a space is absent when defining the MPDs as the search is carried out in three-dimensional space.

MPDs remind of Daudel's loges $[21,22]$ which also use $p_{v}(\Omega)$. There, the idea is to partition molecular space into domains, called "loges", and look for all different possibilities to distribute electrons into them. After some initial trials it was decided to minimize the missing information function,

$$
H\left(x_{1}, \ldots, x_{M}\right)=-\sum_{k} P\left(x_{k}\right) \ln \left(P\left(x_{k}\right)\right)
$$


as the criterion to determine the partition. In the definition of $H$, an event $x_{k}$ is a given distribution of electrons in the loges, $P\left(x_{k}\right)$ is the probability to have such a distribution, and the sum goes over all possible distribution of electrons in the loges. ${ }^{1}$

\subsubsection{Models}

We will treat below some non-interacting particle models for which the exact solution of the Schrödinger equation is known, because it is possible to compute $p_{v}(\Omega)$ for these systems with arbitrary accuracy, e.g., with Mathematica [23]. More details about the models can be found in the appendix.

In order to have a significance for chemistry, we will assume that particles are fermions. Thus, although non-interacting, the particles are not independent, as they have to obey the Pauli principle. Please notice that this holds for same-spin particles, while particles with opposite spin are independent in these models.

In the following, we will sometimes consider situations where only particles of a given spin are present. For $N$ particles of one spin, the probabilities can be related to those for $N$ pairs of fermions of opposite spin. This can be easily seen, by writing the wave function as a product of a Slater determinant for $\alpha$ spin with one for $\beta$ spin (see, e.g. [24]). This product yields the same expectation values as the Slater determinant written with all spin-orbitals, for both spins. Equation 10.1 yields for the probability of finding $v_{\alpha}$ and $v_{\beta}$ electrons in $\Omega$ the product of the probabilities computed for each spin individually, for $v_{\alpha}$, and $v_{\beta}$, respectively. When we consider the restricted Hartree-Fock closed shell form, we obtain the same terms for each spin. Thus, one finds the same $\Omega$ when maximizing the probability of finding $v_{\alpha}$ and $v_{\beta}$ electrons as when one maximizes the probability of finding $v_{\alpha}$ or $v_{\beta}$ electrons only.

\subsection{Results}

\subsubsection{Experience with MPDs}

Up to now, experience has shown that MPD correspond to regions to which a chemist would associate bonds, or cores, or lone pairs (see, e.g. [10]). In this respect, when a single Lewis picture is sufficient, $\Omega_{v}$ s resemble images produced

\footnotetext{
${ }^{1}$ The basis of the logarithm is arbitrary; we have chosen in this paper the natural logarithm, and not the binary logarithm, as usually done.
} 
Fig. 10.1 $\Omega_{10}$ solutions for the $\mathrm{MgO}$ crystal: $\mathrm{Mg}^{2+}$, top, and $\mathrm{O}^{2-}$, bottom

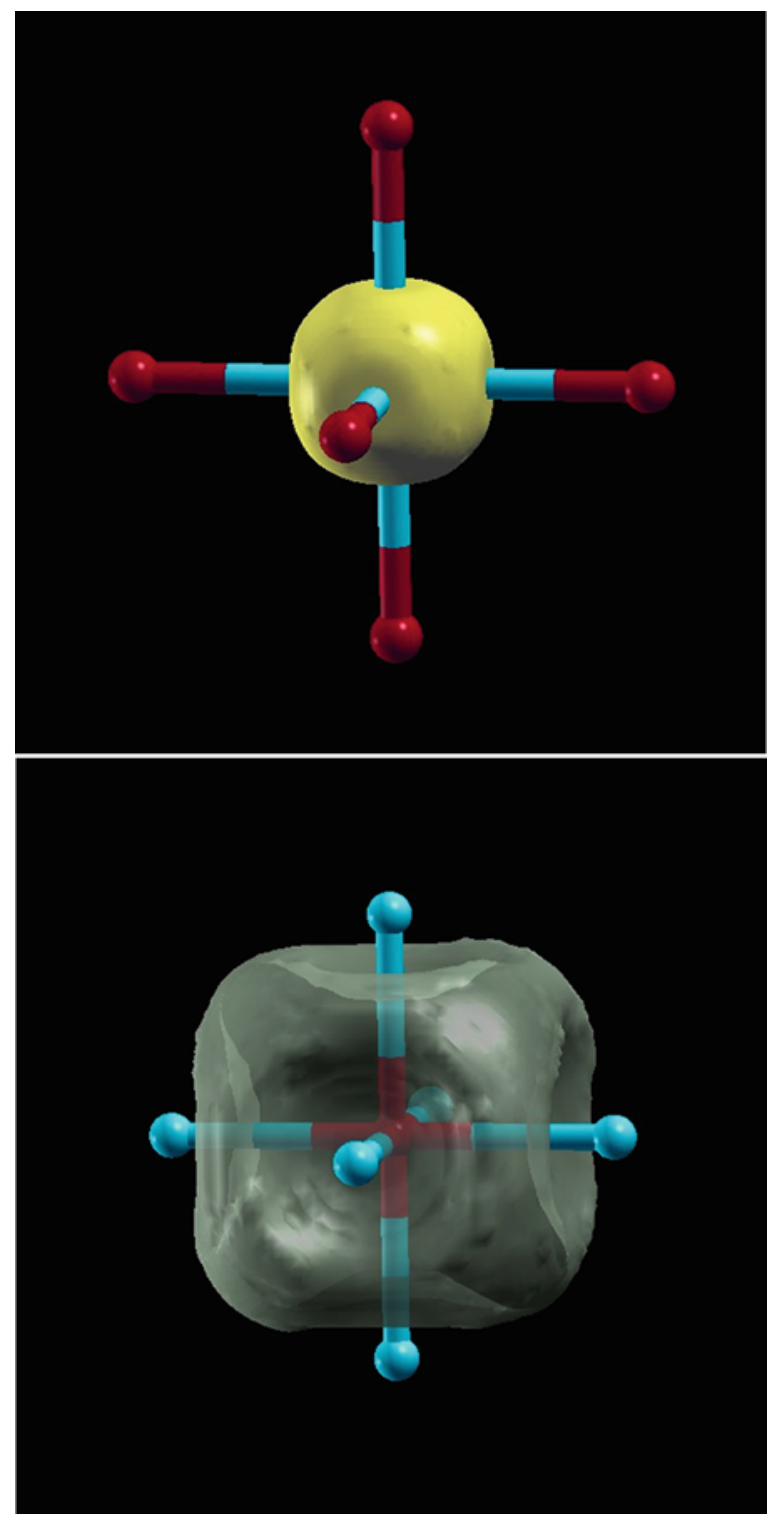

by other tools, e.g., ELF basins $[12,13]$. For example, for the $\mathrm{MgO}$ crystal, when maximizing $p_{10}$, it yields two solutions, one corresponding to $\mathrm{Mg}^{2+}$ and another to $\mathrm{O}^{2-}$, see Fig. 10.1. In fact, it was noticed that the results are slightly closer to chemical intuition than with ELF. For example, with MPDs, the population of atomic shells is closer to the integer numbers one intuitively expects than it is with ELF basins [1]. 


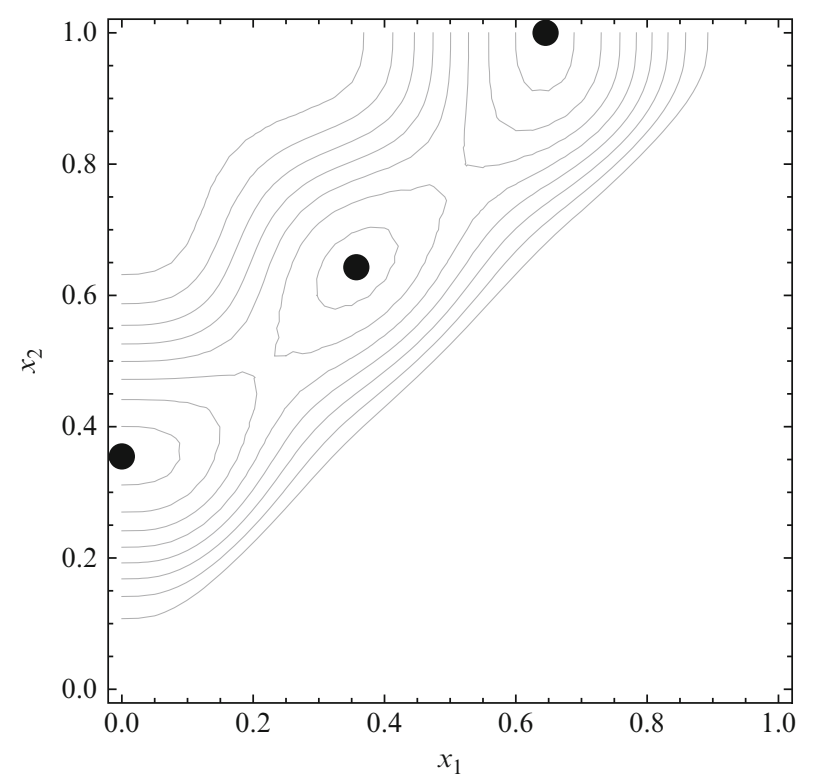

Fig. 10.2 $p_{1}\left(\left[x_{1}, x_{2}\right]\right)$ for three same-spin fermions in a box. The dots mark the maxima

\subsubsection{MPDs Are not Unique}

In general, there can be several MPDs, $\Omega_{v}$, for a given $v$. We just mentioned (cf. Fig. 10.1) that maximizing $p_{10}$ can yield two physically relevant solutions, one corresponding to $\mathrm{Mg}^{2+}$, and another corresponding to $\mathrm{O}^{2-}$. This is physically motivated, and is not a limitation of MPDs. From the practical viewpoint, it means that the program searching for $\Omega_{v}$ can yield several solutions, typically by using different starting guesses.

An accurate calculation showing multiple solutions can be produced for three same-spin particles in a box of unit length. The probability to find one particle in the interval between $x_{1}$ and $x_{2}, p\left(\left[x_{1}, x_{2}\right]\right)$, is shown in Fig. 10.2. There are two maxima corresponding to symmetrically arranged $\Omega$, given by the intervals $[0,0.35]$, at the left of the box, and $[0.65,1]$, at the right of the box. There is a third $\Omega$ between them. It is no surprise that the values for $p_{1}$ are different for the $\Omega_{1}$ at the borders of the box $(\approx 0.84)$ and in the center of the box $(\approx 0.75)$. The lower value for the central $\Omega_{1}$ can be understood by the existence of two penetrable walls for this MPD, while there is only one for the terminal $\Omega_{1}$ s.

The example above also shows that there are solutions which are equivalent, the $\Omega_{1}$ corresponding to the left is equivalent to that on the right border of the box. The origin of this equivalence, is the symmetry of the box. It reminds of the equivalence of localized orbitals [25]: a symmetry operation can transform one localized orbital into another. In a molecule, such equivalent MPDs are common. For example, in 
Fig. 10.3 The two lone pair $\Omega_{2} \mathrm{~S}$ in the $\mathrm{H}_{2} \mathrm{O}$ molecule

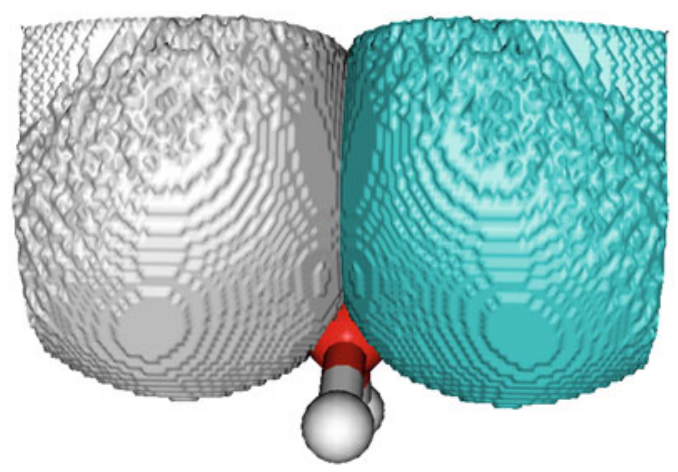

the water molecule, there is a MPD corresponding to one of the lone pairs, that is equivalent to another one, corresponding to the other lone pair, cf. Fig. 10.3.

However, symmetry operations can also produce physically reasonable, equivalent solutions, but not transform one chemically relevant unit (like a bond) into another. In certain situations, there may be an infinite number of equivalent solutions. Take, for example, three same-spin particles in a ring. There is a solution corresponding to $\theta$ between 0 and $\approx 2 \pi / 3$. Of course, there are two more solutions, one corresponding to roughly $[2 \pi / 3,4 \pi / 3]$, and another one for roughly $[4 \pi / 3,2 \pi]$. These are produced from the first domain by threefold rotations. However, the ring is invariant to rotations by an arbitrary angle. Thus, besides the chemically understandable existence of threefold solutions, one can find an infinity of $\Omega_{1}$, as produced by a rotation by an arbitrary angle: $p_{1} \approx 0.68$ does not change when the lower and the upper limit of the interval is displaced by the same arbitrary constant.

A similar result is obtained for acetylene. There is a solution corresponding to one of the banana bonds, see Fig. 10.4. There are two more MPDs, corresponding to the other two banana bonds. One can generate, however, infinitely many new $\Omega_{1}$, by rotating the previous $\Omega_{1}$ s around the internuclear axis. Please notice that the three $\Omega$ produced by the rotation about a threefold axis are essentially non-overlapping. One can see the rotation by an arbitrary angle as the arbitrariness in the choice of the set of three banana bonds.

Sometimes, there only is a finite number of supplementary solutions dictated by symmetry. Let us consider the case of three protons at infinite separation, occupying the vertices of an equilateral triangle. Let us put two electrons of the same spin into this system. One of the degenerate wave functions of the system is given by the Slater determinant:

$$
\Phi_{a}=\left|\frac{1}{\sqrt{3}}\left(\chi_{a}+\chi_{b}+\chi_{c}\right) \frac{1}{\sqrt{6}}\left(2 \chi_{a}-\chi_{b}-\chi_{c}\right)\right|
$$

It can also be written as:

$$
\Phi_{a}=\frac{1}{\sqrt{2}}\left(\left|\chi_{a} \chi_{b}\right|+\left|\chi_{a} \chi_{c}\right|\right)
$$


Fig. 10.4 Two views of an $\Omega_{2}$ corresponding to one of the three banana bonds in acetylene
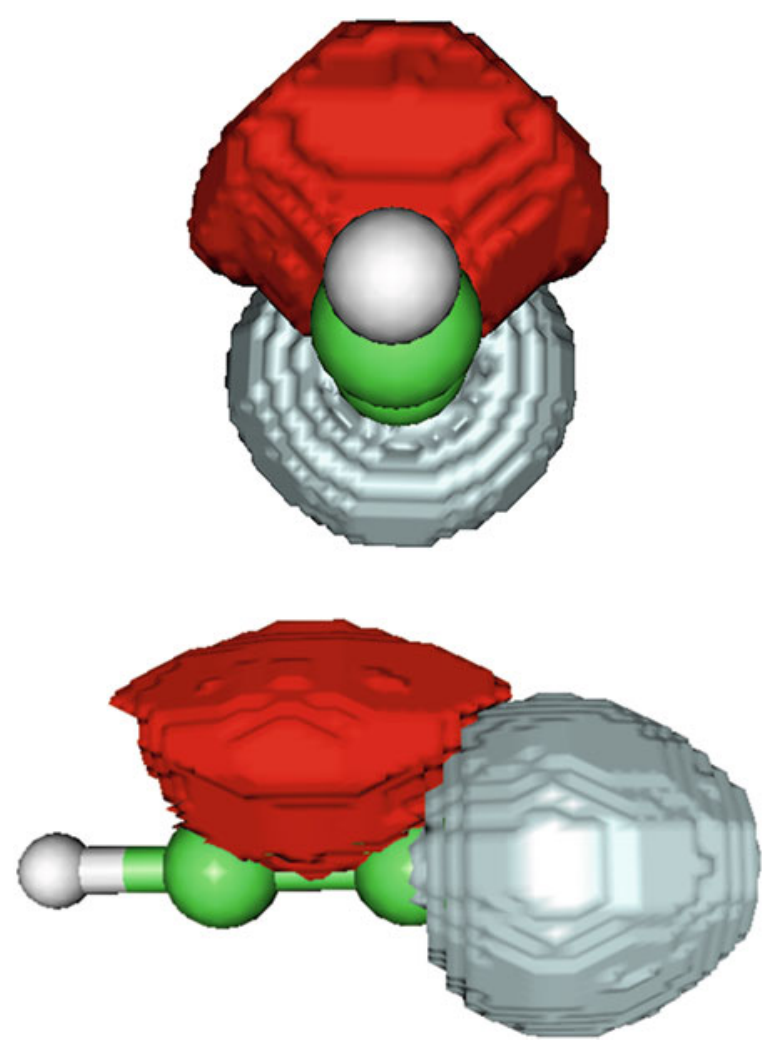

From the latter formulation, one can immediately see that for this wave function, one has $p_{1}=1$ when $\Omega_{1}$ contains either the space associated to one proton, $\mathrm{H}_{a}$, or that for two of them, $\mathrm{H}_{b}$ and $\mathrm{H}_{c}$, cf. Fig. 10.5. One has a system $H \ldots H_{2}^{+}$. This separation can be done, of course, also by isolating $\mathrm{H}_{b}$, or $\mathrm{H}_{c}$. These solutions can be obtained by a rotation along the threefold axis. Notice that there are three solutions, but as there are only two electrons of same spin these solutions overlap significantly, and do not form a partition of space, as it is given by $H \ldots H_{2}^{+}$. Symmetry thus provides alternative chemically equivalent solutions.

A similar situation has been noticed for the $\mathrm{Si}_{2} \mathrm{H}_{2}$ molecule, in $D_{2 h}$ symmetry (bent acetylene structure) [10]. One also obtains MPDs corresponding to banana bonds. By the bending, however, the $C_{\infty}$ axis which was present in acetylene is not present anymore, so that an arbitrary rotation around the $\mathrm{Si}$-Si axis does not produce an equivalent solution. The molecule still has inversion symmetry. By inversion, the arrangement of the three banana bond like MPDs, in the $\triangle$ arrangement, are transformed into one having a $\nabla$ arrangement. 


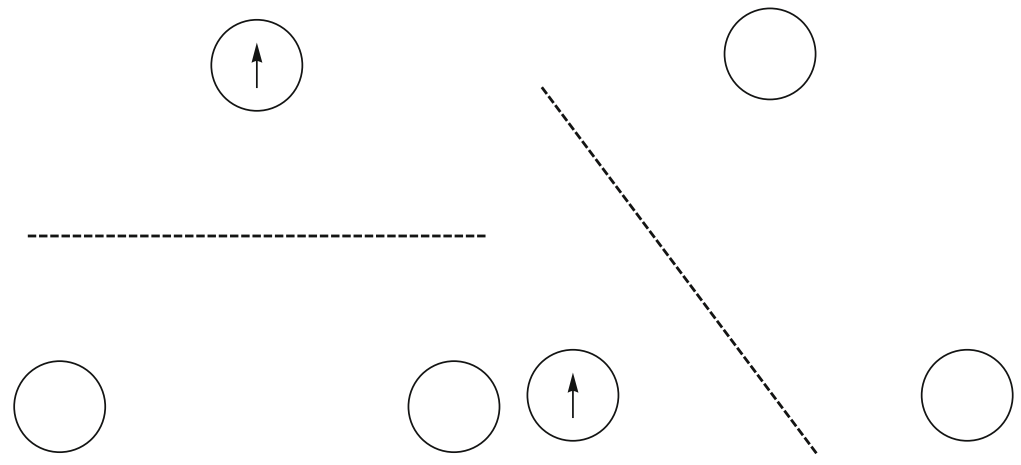

Fig. 10.5 Two choices leading to $\Omega_{1}$ with $p_{1}=1$ for three protons and two same-spin electrons

\subsubsection{MPDs do not Always Provide an Exact Partition of Space}

In the ground state of two non-interacting same-spin fermions in a one-dimensional box, $\Omega_{1}$ corresponds to exactly one half of the box. The two solutions for $\Omega_{1}$ thus provide a partition of the box. In general, however, MPDs do not necessarily provide an exact partition of space. For example, small overlaps were noticed in some molecular calculations $\left(\mathrm{CH}_{5}^{+}\right.$or $\mathrm{FHF}^{-}$[10]). However, the discretization by small cubes, and numerical noise left a question mark with this statement. In order to clarify whether MPDs necessarily provide a partition of space, we consider now a model, for which we can state with certitude that MPDs do not provide a partition of space. We choose again the example of the three non-interacting fermions of the same spin in a one-dimensional box. The $\Omega_{1}$ s are given by: $0 \leq x \leq 0.3547$, $0.3572 \leq x \leq 0.6428$, and $0.6453 \leq x \leq 1$. Small regions $0.3547<x<0.3572$ and $0.6428<x<0.6453$ have not been attributed. Similarly, for the three electrons on a ring, $\Omega_{1}$ is not delimited by 0 and exactly $2 \pi / 3$; the upper limit is $\approx 2.06<2 \pi / 3$. With the present accuracy of our programs, such small effects could not yet be detected with certainty in molecular or crystalline systems.

\subsubsection{MPDs can be Disjoint in Space}

We have seen that for the ground state of two same-spin non-interacting fermions in a box, the $\Omega_{1}$ correspond to half of the box, with $p_{1} \approx 0.86$. In the excited states, this is not the case anymore. For example, if one fermion is excited from the $n=2$ to the $n=3$ state, there is a solution for $\Omega_{1}, x \in[0.32,0.62]$ with $p_{1} \approx 0.88$. There is another solution for $\Omega_{1}$, with the same $p_{1}$, for the rest of the box, $x \in[0,0.32] \cup$ $[0.62,1]$. For it, a description with a single basin is not possible. This is a situation that does not correspond to the classical image of localized electrons, but shows nevertheless a high probability. Such a situation can appear in resonating systems (when several Lewis structures are needed). 


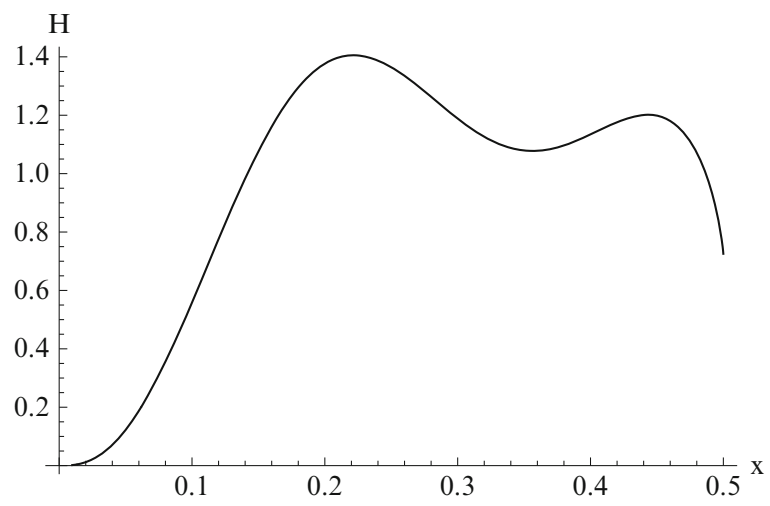

Fig. 10.6 Missing information function $H$ for loges defined by the intervals $[0, x],(x, 1-x),[1-$ $x, 1]$, as a function of $x$

\subsubsection{MPDs and Loges}

In this section we will highlight some differences between MPDs and loges. Let us consider again the system of three same-spin non-interacting particles in a box. We define the loges by the intervals $[0, x],(x, 1-x),[1-x, 1]$. The missing information function $H$, Eq. 10.4, depends on $x$, see Fig. 10.6. $H$ presents a minimum, for $x \approx$ 0.3565 , close to the value obtained by maximizing $p_{1}$. This is a local minimum, and there are two other, lower minima. The first one, at $x=0$ is trivial. It corresponds to making the border loges vanish, keeping only the central loge. It is a minimum of $H$, as the probability of finding all three electrons in the whole box is equal to 1 . This situation can be easily identified and discarded. There is another minimum, however, for $x=1 / 2$ taking a lower value than for the physically interesting minimum. It is the case where the central box vanishes, and only the distributions where there is no electron in the central box contribute to $H$. Please notice that taking $x<1 / 2$, but close to this value, the central loge has not vanished yet, but $H$ takes a low value. Thus, it is not easy to detect such a situation when minimizing $H$.

\subsection{Conclusions}

In order to better understand the maximum probability domains (the region of space maximizing the probability to find a given number of electrons in it), we studied some simple model systems.

Electronic systems well described by a single Lewis structure produce MPDs which correspond roughly to a partition of space which permits an association to bonds, lone pairs, or cores. However, our models have shown that this partition is not strict. A further point is that symmetry can produce alternative solutions. 
The model calculations have proven that MPDs formed by disjoint subdomains can exist.

It was shown that using the missing information function, as it is done in loge theory, can produce unwanted results.

\subsection{Appendix: Detailed Description of the Models}

Let us now describe the simple models used in this paper. The first one is that of non-interacting particles in a one-dimensional box. We will choose the box to have unit length, and the orbitals are given by:

$$
\varphi_{n}(x)=\sqrt{2} \sin (n \pi x)
$$

The overlaps for $\Omega$ defined by the interval $\left[x_{1}, x_{2}\right]$ is given by

$$
S_{i j}\left(\left[x_{1}, x_{2}\right]\right)=\int_{x_{1}}^{x_{2}} \varphi_{i}(x) \varphi_{j}(x) d x
$$

and easily computed and diagonalized, yielding the eigenvalues $\lambda_{i}$. For two fermions of the same spin the probability to find one electron in this interval is given by [3]

$$
p_{1}\left(\left[x_{1}, x_{2}\right]\right)=\lambda_{1}+\lambda_{2}-2 \lambda_{1} \lambda_{2}
$$

A slightly more complicated expression is obtained for three particles of the same spin.

For particles in a ring, the orbitals are given by

$$
\varphi_{k}(\theta)=\frac{1}{\sqrt{2 \pi}} e^{i k \theta}
$$

for $\theta$ between 0 and $2 \pi$.

For three infinitely distant hydrogen atoms, with the nuclei on the vertices of an equilateral triangle, the orbitals are:

$$
\begin{aligned}
& \varphi_{1}=\frac{1}{\sqrt{3}}\left(\chi_{a}+\chi_{b}+\chi_{c}\right) \\
& \varphi_{2}=\frac{1}{\sqrt{6}}\left(2 \chi_{a}-\chi_{b}-\chi_{c}\right) \\
& \varphi_{3}=\frac{1}{\sqrt{6}}\left(-\chi_{a}+2 \chi_{b}-\chi_{c}\right) \\
& \varphi_{4}=\frac{1}{\sqrt{6}}\left(-\chi_{a}-\chi_{b}+2 \chi_{b}\right)
\end{aligned}
$$


where only two of $\varphi_{2}, \varphi_{3}, \varphi_{4}$ are linearly independent. Please notice that $S(\Omega)$ is simplified as all products between the functions centered on different atoms are vanishing.

\section{References}

1. Savin A (2002) In: Sen KD (ed) Reviews of modern quantum chemistry: a celebration of the contributions of Robert G. Parr. World Scientific, Singapore, p 43

2. Chamorro E, Fuentealba P, Savin A (2003) J Comp Chem 24:496

3. Cancès E, Keriven R, Lodier F, Savin A (2004) Theor Chem Acc 111:373

4. Gallegos A, Carbó-Dorca R, Lodier F, Cancès E, Savin A (2005) J Comp Chem 26:455

5. Francisco E, Martín Pendás A, Blanco MA (2007) J Chem Phys 126(9):094102

6. Martín Pendás A, Francisco E, Blanco MA (2007) J Chem Phys 127:144103

7. Martín Pendás A, Francisco E, Blanco MA (2007) Chem Phys Lett 437:287

8. Martín Pendás A, Francisco E, Blanco MA (2007) Phys Chem Chem Phys 9:1087

9. Martín Pendás A, Francisco E, Blanco MA (2007) J Phys Chem A 111:1084

10. Scemama A, Caffarel M, Savin A (2007) J Comp Chem 28:442

11. Francisco E, Martín Pendás A, Blanco MA (2009) J Chem Phys 131:124, 125

12. Silvi B, Savin A (1994) Nature 371:683

13. Becke A, Edgecombe KE (1992) J Chem Phys 92:5397

14. Noury S, Krokidis X, Fuster F, Silvi B (1999) Comput Chem 23:597

15. Scemama A (2005) J Theor Comp Chem 4:397

16. Frisch MJ, Trucks GW, Schlegel HB, Scuseria GE, Robb MA, Cheeseman JR, Scalmani G, Barone V, Mennucci B, Petersson GA, Nakatsuji H, Caricato M, Li X, Hratchian HP, Izmaylov AF, Bloino J, Zheng G, Sonnenberg JL, Hada M, Ehara M, Toyota K, Fukuda R, Hasegawa J, Ishida M, Nakajima T, Honda Y, Kitao O, Nakai H, Vreven T, Montgomery JA Jr, Peralta JE, Ogliaro F, Bearpark M, Heyd JJ, Brothers E, Kudin KN, Staroverov VN, Kobayashi R, Normand J, Raghavachari K, Rendell A, Burant JC, Iyengar SS, Tomasi J, Cossi M, Rega N, Millam JM, Klene M, Knox JE, Cross JB, Bakken V, Adamo C, Jaramillo J, Gomperts R, Stratmann RE, Yazyev O, Austin AJ, Cammi R, Pomelli C, Ochterski JW, Martin RL, Morokuma K, Zakrzewski VG, Voth GA, Salvador P, Dannenberg JJ, Dapprich S, Daniels AD, Farkas Ö, Foresman JB, Ortiz JV, Cioslowski J, Fox DJ (2009) Gaussian 09 revision A.1. Gaussian Inc., Wallingford CT

17. Saunders VR, Dovesi R, Roetti C, Orlando R, Harrison NM, Zicovich-Wilson CM (1998) CRYSTAL98 (CRYSTAL98 user's manual). University of Torino, Torino

18. Francisco E, Pendás AM, Blanco MA (2008) Comput Phys Commun 178:621

19. Bader RFW (1990) Atoms in molecules: a quantum theory. Oxford University Press, Oxford

20. Savin A (2005) J Chem Sci 117:473

21. Daudel R (1953) CR Acad Sci France 237:691

22. Aslangul C, Constanciel R, Daudel R, Kottis P (1972) Adv Quantum Chem 6:93

23. Wolfram S (2008) Mathematica edition: version 7.0. Wolfram Research, Inc., Champaign

24. Wigner E (1934) Phys Rev 46:1002

25. Lennard-Jones J, Pople JA (1950) Proc Roy Soc A 202:166 